\title{
AXL phosphorylates and up-regulates TNS2 and its implications in IRS-1-associated metabolism in cancer cells
}

\author{
Li-Chun Cheng ${ }^{1,3}$, Yen-Lin Chen ${ }^{2}$, An-Ning Cheng ${ }^{3}$, Alan Yueh-Luen Lee ${ }^{3}$, Chun-Yu Cho ${ }^{3}$, Jhy-Shrian Huang ${ }^{4}$ \\ and Shuang-En Chuang ${ }^{1,3^{*}}$
}

\begin{abstract}
Background: TNS2 is a focal adhesions protein and a binding partner for many proteins, including the receptor tyrosine kinase Axl. Although TNS2 can bind with Axl, the details of their interactions have not been elucidated. TNS2 is involved in IRS-1 signaling pathway. In this study, we confirmed the relationship between TNS2 expression and the expression of Axl, IRS-1, PDK1 and Glut4 in pancreatic cancer patients.

Methods: The expression levels of TNS2, AxI, IRS-1, PDK1 and Glut4 in human cancer cells were measured by Western blot and/or IP-Western blot assays. Paired samples of pancreatic cancer and non-cancer tissues were obtained from 33 patients and were used to construct tissue microarrays. The expression levels of these markers in the tissue microarrays were measured by enzyme-linked Immunohistochemistry assay, and the relationships were analyzed by Pearson's chi-square test and two-tailed t-test analysis.

Results: We demonstrated for the first time that TNS2 is a phosphorylation substrate of AxI. Moreover, we found a positive relationship between TNS2 expression and the expression of Axl, IRS-1, PDK1 and Glut4 in pancreatic cancer patients. Based on these results, we suggest that Axl modulates glucose metabolism potentially through TNS2 and IRS-1. We hypothesize that there exists a novel mechanism whereby Axl binds to and phosphorylates TNS2, releasing TNS2 from interaction with IRS-1 and resulting in increased stability of IRS-1. The two key enzymes of aerobic glycolysis (Glut4 and PDK1) were found to be up-regulated by AxI/TNS2/IRS-1 cross-talk and may play a critical role in glucose metabolism of cancer cells.
\end{abstract}

Conclusions: Our results revealed for the first time that Axl binds to and phosphorylates TNS2 and that Axl/TNS2/ IRS-1 cross-talk may potentially play a critical role in glucose metabolism of cancer cells.

Keywords: Axl, Tensin2, C1-TEN, IRS1, PDK1, Glut4, Pancreatic cancer, Glucose metabolism

\section{Background}

Axl is a receptor tyrosine kinase, which is characterized as a pro-oncogenesis factor involved in cancer progression. Along with its ligand Gas6, Axl, through its signaling, plays important physiological roles in the nervous [1], reproductive, and vascular systems [2]. Axl is also known to be associated with hemostasis, inflammation, autoimmune diseases [1-3] and pancreatic cancer $[4,5]$.

\footnotetext{
*Correspondence: sechuang@nhri.org.tw

'Graduate Institute of Life Sciences, National Defense Medical Center, Taipei, Taiwan

${ }^{3}$ National Institute of Cancer Research, National Health Research Institutes, 35 Keyan Road, Zhunan, Miaoli County 350, Taiwan, Republic of China Full list of author information is available at the end of the article
}

At high-glucose conditions, Axl binds to and conveys signals through phosphatidylinositol-3-kinase (PI3K), resulting in apoptosis of vascular smooth muscle cells. At low-glucose conditions, Axl binds to the protein tyrosine kinase SHP2; this results in increased cell migration (diabetic cardiovascular disease) and survival (anti-apoptosis) [2]. However, whether Axl is involved in cancer metabolism for cancer progression remains largely unknown. In this study, for the first time, we demonstrate that Axl may affect glucose metabolism through TNS2.

Tensin2 - also known as TNS2, TENC1, and C1-TEN - is a member of the Tensin family that also includes tensin 1 , tensin 3 and tensin4/CTEN. All four members 
can be found in focal adhesions and fibrillar adhesions, and they can modulate both cell motility and transformation [6]. TNS2 contains a Src Homology 2 (SH2) domain and a phosphotyrosine-binding (PTB) domain, both of which allow it to interact with tyrosine-phosphorylated proteins such as the PI3K/Akt kinase, p130Cas, and focal adhesion kinase (FAK) [6, 7]. It is unclear whether TNS2 is regulated by these tyrosine-phosphorylated proteins. TNS2 has been identified as a binding partner for several different kinases and proteins, including Axl $[8,9]$, the non-receptor tyrosine kinase Syk [10], thrombopoietin receptor Mpl [11], insulin receptor substrate 1 (IRS-1) [12], deleted in liver cancer 1 (DLC1) [13-15], and disrupted in schizophrenia 1 (DISC1) [16]. It is plausible that TNS2 conveys different signals by binding with different partners involved in cancer progression. For instance, the tumor suppressor DLC1 may interact with TNS2 and contribute to the growth-suppressive activity of DLC1 in hepatocellular carcinoma [13]. Silencing TNS2 expression may increase the activities of Akt, Mek, and IRS-1 in lung and cervical cancers [17]. However, Jung et al. [11] demonstrated that another consequence of TNS2 binding to $\mathrm{Mpl}$ is recruitment of PI3K to the plasma membrane, where it can phosphorylate PIP2, thereby activating the downstream effector $\mathrm{AKT} / \mathrm{PKB}$ in platelet disorders. The role of TNS2 in cancer remains a mystery. TNS2 interacts not only with different binding partners but also with different isoforms. TNS2 has three different isoforms, variant 1 to 3 (V1, V2, and V3). High expression of TNS2 V1 and V3 has been found in skeletal muscle, whereas TNS2 V2 has been found in pancreas and heart [17]. In this study, we examined the signaling effects of the TNS2 V2 isoform in pancreatic cancer cell lines.

Although a previous study found that TNS2 may bind with Axl in yeast two-hybrid interactions [8], the details of the interaction remain to be elucidated. In our research, we demonstrate that TNS2 serves as a substrate for Axl and may play a critical role in cancer by modulating Axl signaling. The pancreas secretes insulin and glucagon, two of the hormones that regulate glucose metabolism. Notably, pancreatic tumors heavily rely on glycolysis [18]. Therefore, we further examined the correlation of TNS2 expression and expression of Axl, IRS-1, pyruvate dehydrogenase kinase 1 (PDK1) and glucose transporter type 4 (Glut4) in tissue of 33 patients with pancreatic cancer. PDK1 is a critical determinant of glucose metabolism and Warburg-like metabolic changes (or aerobic glycolysis) $[19,20]$. Our results suggest that Axl binds to and phosphorylates TNS2 and is involved in glucose metabolism in human pancreatic cancer cells.

\section{Methods}

\section{Cell culture and treatment}

HEK293T, human osteosarcoma U2OS cells and human pancreatic cnacer Panc-1 cells were grown in DMEM supplemented with $10 \%$ fetal bovine serum (FBS) and $1 \%$ penicillin/streptomycin (Invitrogen, USA). Mia $\mathrm{PaCa}-2$ cells were cultured in DMEM supplemented with $2.5 \%$ horse serum, $10 \%$ FBS and $1 \%(w / v)$ penicillin/streptomycin (Invitrogen, USA). Cells were routinely grown in a humidified incubator with $5 \% \mathrm{CO}_{2}$ at $37^{\circ} \mathrm{C}$. Following transfection with various plasmids for $24 \mathrm{~h}$, cells were treated with/without $400 \mathrm{ng} / \mathrm{mL}$ recombinant human Gas6 (R\&D Systems Inc., Minneapolis, MN) for $15 \mathrm{~min}$. The cells were then harvested and analyzed for gene expression by Western blot. All these cell lines were authenticated by Mission Biotech (Taipei, Taiwan) and DNA typing was analyzed with ABI PRISM 3730 Genetic Analyzer and GeneMapper software V3.7.

\section{Plasmid constructs}

The Axl recombinant plasmid and shRNA expression vecter constracts were followed in our previous publish [21]. Wild-type human Tensin2 plasmid (KIAA1075), which was cloned into pCMV-Myc mammalian expression vector, was a gift from Drs. B. Dahlbäck and S. Hafizi. A series of mutation variants were cloned into the pCDNA3.0 vector (Novagen, Australia). The site-directed mutagenesis for the generation of Myc-TNS2 (C231S) and Myc-TNS2 (Y483S) was performed with a KOH Hotstar DNA polymerase kit (Merck Millipore, Darmstadt, Germany) using the following primers:

\section{5' - GGAT GGC AGTCCTTTTGCCCAGGTGCAGC G-3' and 5'-CGCTGCACCTGGGCAAAAGGACTGCCATCC-3' (for Y483F); and 5'-GTGGTCGTACTATACAGCAAGGGAAACAA GGGC-3' and 5'-GCCCTTGTTTCCCTTGCTGTATAGTACGACC AC-3' (for C231S)}

\section{Immunoprecipitation}

Cells were transfected as aforementioned and grown in 10-cm dishes. To extract proteins, cells were washed with ice-cold PBS and lysed in NETN lysis buffer (150 mM NaCl, $1 \mathrm{mM}$ EDTA, $20 \mathrm{mM}$ Tris-Cl pH 8.0, $0.5 \% \mathrm{NP}-40(\mathrm{v} / \mathrm{v})$ containing protease and phosphatase inhibitors ( $50 \mathrm{mM} \mathrm{NaF}, 0.1 \mathrm{mM} \mathrm{Na} \mathrm{NO}_{3} \mathrm{VO}$ ). The lysates were incubated, respectively, with $2 \mu \mathrm{g}$ of IP antibodies to Myc (9E10) (\#05419, Millipore) or Axl (C-20) (\#sc-1096, Santa Cruz) at $4{ }^{\circ} \mathrm{C}$ overnight with constant rotation. The immunocomplex was captured by incubating with $40 \mu \mathrm{l}$ protein A/G-agarose beads (Sigma Aldrich, Sr. Louis, MO, USA) for $3 \mathrm{~h}$ at $4{ }^{\circ} \mathrm{C}$ with constant rotation. Following $1 \mathrm{~min}$ centrifugation at $10000 \mathrm{~g}$, the beads were washed three times with ice-cold NETN buffer and ready for SDS-PAGE analysis or kinase assay. Representative Western blot results are 
shown in figures and experiments were performed in triplicate.

\section{Immunoblotting}

Cell extracts were subjected to Western blot analysis as previously described [21]. The monoclonal antibody against the Myc-epitope (9B10) was from Millipore Technology. The expression vector of TNS2 was Myc-tagged and cloned into the pcDNA3.0 plasmid. To generate an antibody against $\mathrm{p}$-Axl, the synthetic phospho-oligopeptide

Asp-Gly-Leu-(phospho)Tyr-Ala-Leu-Met-Ser-Arg-Cys was used as an antigen for the generation of rabbit immune serum using a commercial service (GenTex Inc., California). The following antibodies were used: Axl (C-20), Erk sc-94, Santa Cruz), p-Erk (sc-7383, Santa Cruz), Akt 1/2/3 (\#9272, Cell signaling) and p-Akt (\#4051, Cell signaling), IRS-1 (\#06-248,Millipore,), IRS-2 (\#06-506, Upstate), TNS2 (SAB4200268, Sigma Aldrich), pTNS2-Y483 (ab138414, Abcam, Eugene), PDK1 (\#21005, SAB signalway) and Glut4 (\#2231S, Cell signaling).

\section{In vitro kinase assay}

The active His-tagged human recombinant Axl kinase (473-end) was produced from insect Sf9 cells and purchased from SignalChem Life Sciences (Richmond, CA). HEK293T cells were transfected with Tensin2 expression vector and immunoprecipitated for $24 \mathrm{~h}$. The active Axl kinase and Tensin 2 proteins (as substrates) immunoprecipitated from the transfected HEK293T cells were incubated with $10 \mu \mathrm{Ci}\left[\gamma-32^{\mathrm{P}}\right]$ ATP, phosphatase inhibitors (10 mM NaF, $50 \mathrm{mM} \beta$-glycerophosphate) in kinase buffer (25 mM HEPES pH 7.5, $50 \mathrm{mM} \mathrm{NaCl}, 10 \mathrm{mM}$ $\mathrm{MgCl}_{2}, 1 \mathrm{mM}$ DTT, $10 \mu \mathrm{M}$ ATP) at $30{ }^{\circ} \mathrm{C}$ for $40 \mathrm{~min}$, followed by adding SDS-PAGE sample buffer to stop the reaction. Phosphorylated radioactive proteins were separated by SDS-PAGE and detected by autoradiography. R428 is a specific Axl inhibitor. Representative results are shown in Fig. 3c and experiments were performed in triplicate.

\section{Glucose uptake assay}

For the basal glucose uptake assay, U2OS and Mia $\mathrm{PaCa}-2$ cells were seeded in 6 -well plates $\left(5 \times 10^{5}\right.$ cells per well) and cultured for $24 \mathrm{~h}$, washed with phosphate-buffered saline (PBS) and incubated for $3 \mathrm{~h}$ with serum-free medium. All cells were transfected with pcDNA3.0 vector (vehicle) or Tensin2 expression plasmid with or without Axl expression plasmid for $24 \mathrm{~h}$ and then washed with PBS and incubated for $3 \mathrm{~h}$ with serum-free medium. Glucose uptake was measured using a glucose uptake cell-based assay kit (Cayman Chemical, MI) according to the manufacturer's instructions. Three replicates were performed for each group and the experiments were repeated three times to confirm the results. The BD Bioscience FACSCalibur flow cytometry was used for glucose uptake analysis.

\section{Colony formation}

Mia PaCa-2 cells were seeded in a 6-well dish (100 cells per well) overnight, then transfected with various expression vectors as indicated and allowed to grow for $11 \mathrm{~d}$ in a humidified incubator with $5 \% \mathrm{CO}_{2}$ at $37{ }^{\circ} \mathrm{C}$. Representative colony results are shown in figures and experiments were performed in replicate.

\section{Patients and tissue microarrays}

The tissue microarrays were constructed using formalin -fixed, paraffin-embedded archival tissue blocks from 33 patients who had undergone pancreaticoduodenectomy at Cardinal Tien Hospital between 2006 and 2013. We excluded patients who had received chemotherapy or radiation therapy before resection. All patient clinical charts and histopathology reports were reviewed for tumor size, extracapsular invasion, lymph node metastasis or distant metastasis, and TMN stage. For each patient, 2 cores of tumor and 2 cores of paired non-neoplastic pancreatic tissue were sampled from representative areas using a $2.0-\mathrm{mm}$ punch. Due to the heterogeneity of the immunohistochemical staining patterns that are associated with adenocarcinoma, we determined tissue microarray slides via standard hematoxylin and eosin (H\&E) staining and inserted the samples into recipient paraffin blocks to form complete tissue arrays. For each sample selected on an array slide, the carcinoma type, degree of cell differentiation, growth pattern, tumor cell nuclear morphology, degree of metaplasia, degree of calcification, extent of necrosis, mitosis count, and other specific indices of differentiation were rechecked by two pathologists. Sections of $4 \mu \mathrm{m}$ were cut from these complete array blocks and transferred to salinized glass slides. All procedures involving patient tissues were delinked anonymously for protection and approved by the Institutional Review Board (IRB) of Cardinal Tien Hospital (CTH-101-3-5-054).

\section{Immunohistochemical analysis for gene expression}

IHC staining was performed using a Ventana BenchMark XT automated stainer (Ventana, Tucson, AZ). The sections were incubated respectively with various antibodies against Akt 1/2/3, p-Akt 1/2/3, Erk, p-Erk, IRS-1, PDK1, PKM2, and TNS2 for $1 \mathrm{~h}$ at room temperature. For Axl, p-Axl and p-TNS2 detection, the sections were incubated overnight at $4{ }^{\circ} \mathrm{C}$ with anti-AXL (1:50, ab72069, Abcam, Eugene, OR), anti-pAxl-Y779 (1:100 AF2228, R\&D, MN, USA) and anti-p-TNS2 (1:100, ab138414, Abcam, Eugene, OR), 
A

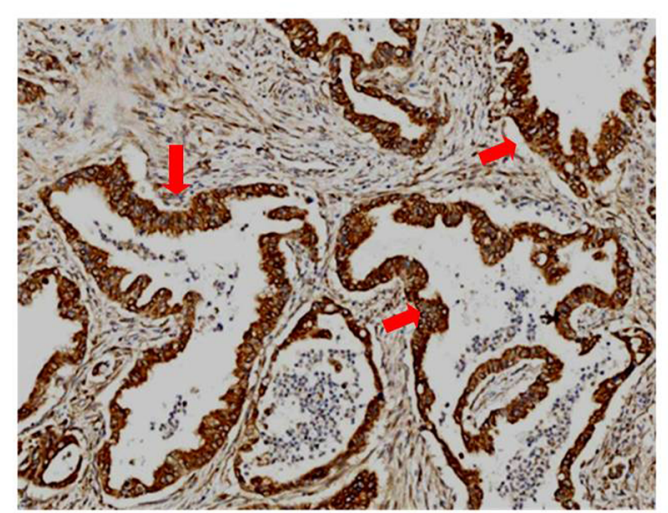

TNS2

Axl

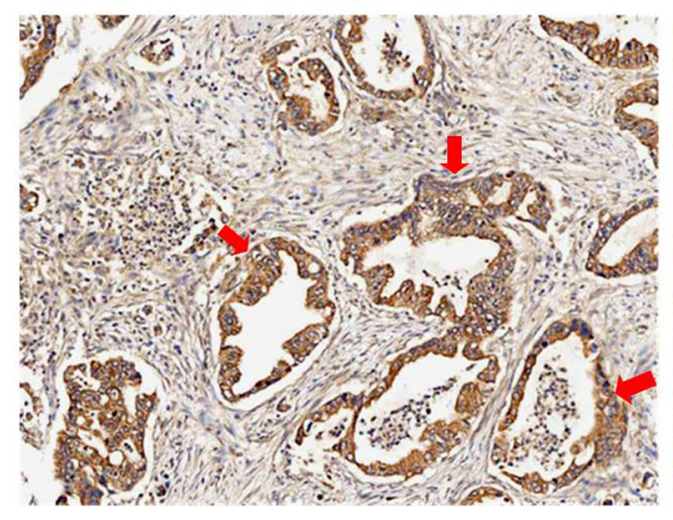

$\begin{array}{lcccc}\text { B Mia pa2 } & \underset{2 u g}{V} & \frac{T N S 2}{2 u g} & \text { 4ug } & \frac{\text { TNS2 }}{4 \text { ug }}\end{array}$
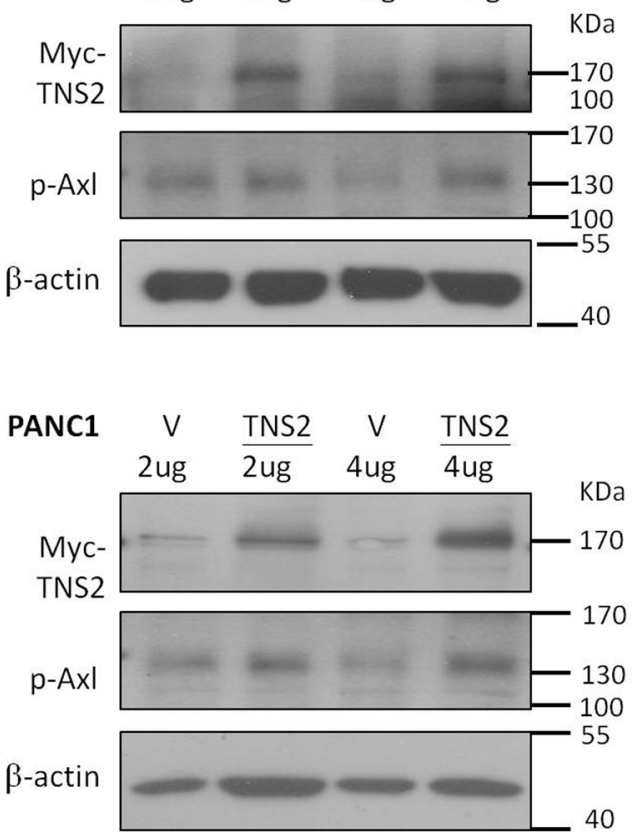

pTNS2

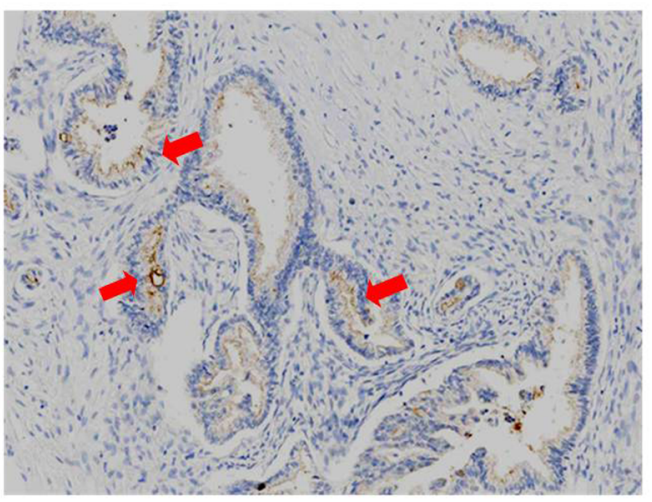

$\mathrm{pAxl}$

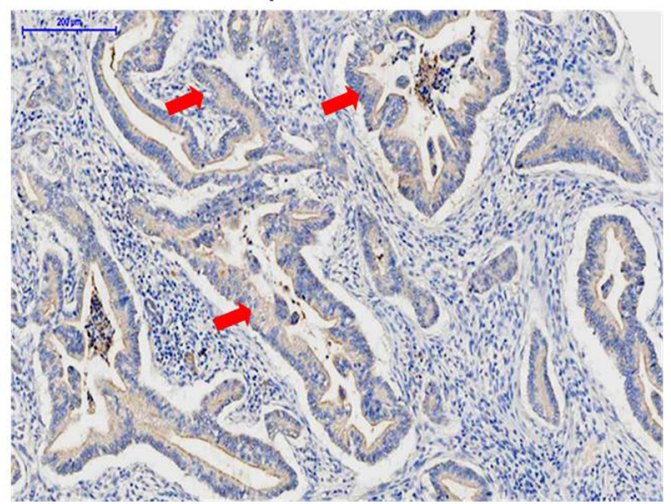

C

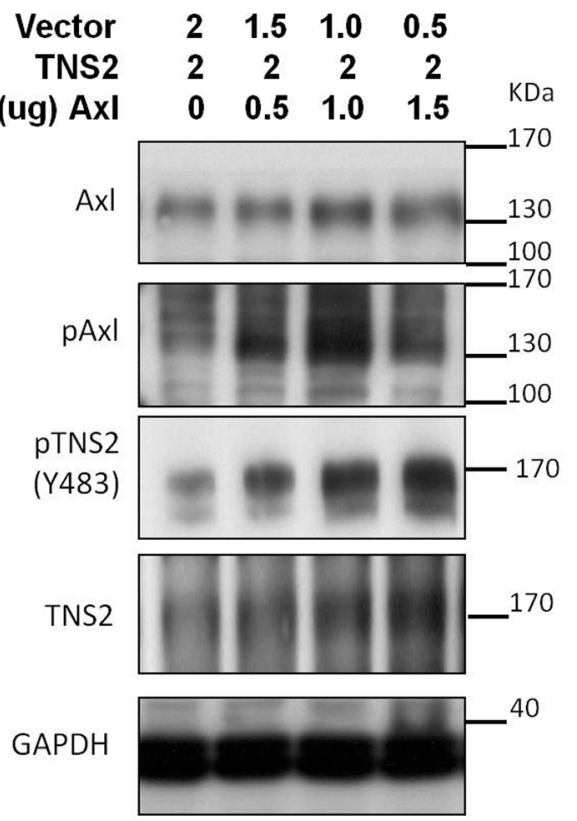

Mia paca2

Fig. 1 (See legend on next page.) 
(See figure on previous page.)

Fig. 1 Expression of TNS2 and Axl in pancreatic cancer. a Representative micrographs show the histopathologic feature and the expression of TNS2 and AXL in 33 PDAC tumor tissues by IHC. Scale bar, $200 \mu \mathrm{m}$ (b) Mia PaCa2 and PANC1 pancreatic cancer cells were transfected with the Myc-tagged wild type TNS2 expression plasmid or the empty vector control pcDNA3 and analyzed by Western blot $24 \mathrm{~h}$ later. c Mia PaCa2 cells were transfected with Axl or TNS2 or both followed by Western blot analysis $24 \mathrm{~h}$ later

respectively. Antibody binding was revealed using the Strepta ABComplex/HRP Duet kit (Dako, Santa Barbara, CA, USA). To evaluate Axl and other protein expression in pancreatic ductal adenocarcinoma (PDAC) cells and non-neoplastic pancreatic epithelial cells, the IHC staining was rechecked by two pathologists. The immunostaining results were scored quantitatively based on staining intensity and the percentage of positively stained cells. An $\mathrm{H}$-score ranging from 0 to 300 was calculated by multiplying the staining intensity score ( 0 for no staining, 1 for faint staining, 2 for moderate staining, and 3 for strong staining) by the percentage of positively stained cells (ranging from 0 to 100). PDAC cases were categorized into 3 groups based on $\mathrm{H}$-score: low expression $(\mathrm{H}$-score 0-100), moderate expression ( $\mathrm{H}$-score 101-200), and high expression (H-score 201-300). The immunohistochemically stained slides of PDAC tissue microarrays were scanned at $200 \times$ magnification with a Pannoramic MIDI II digital slide scanner (3DHISTECH, Budapest, Hungary).

\section{Statistical analysis}

The densitometric quantification of Western blot was performed by Image J and analyzed by t-test. Gene expression in PDAC and adjacent non-tumor tissues was analyzed by Pearson's chi-square statistics. The correlation between different genes in PDAC cancer samples were analyzed by two-tailed t-test analysis. All differences were considered statistically significant when $p$-value $<0.05$.

\section{Results}

\section{Expression of TNS2 and Axl in pancreatic cancer}

TNS2 has been demonstrated to bind with Axl [8] and down regulates Akt expression [22]. The TNS2 expression status of five pancreatic cancer cell lines was determined by Western blot analysis. While AsPC1, HPDE, and SUIT-2 expressed little TNS2, the two relatively drug-resistant cell lines, Mia PaCa-2 and PANC1, were found to express higher levels of TNS2 (Additional file 1). In the tumor tissue microarray, high expression levels (H-score 201-300) of TNS2, p-TNS2, Axl, and p-Axl were detected in the pancreatic ductal areas of 33 PDAC patients' tumor tissues (Fig. 1a). Ectopic expression of TNS2 did not significantly alter the levels of p-Axl (Fig. 1b). When Axl and the Myc-tagged TNS2 expression constructs were co-transfected into Mia $\mathrm{PaCa}-2$ cells, the levels of both TNS2 and p-TNS2 were significantly increased by $\mathrm{Axl}$ transfection in a dose-dependent manner (0 to $1.5 \mu \mathrm{g}$ of Axl plasmid DNA) (Fig. 1c).

\section{Axl knockdown abrogates TNS2 expression and down- regulates its downstream signaling and cellular glucose import}

When Myc-TNS2 and Axl were co-transfected into the non-Axl-expressing HEK293T cells, p-Axl expression was markedly higher in co-transfected cells compared with that in cells with Myc-TNS2 alone or Axl alone (Fig. 2a). Similar results were observed for the levels of p-Erk but not for the levels of p-Akt, both of which are Axl downstream molecules. (Fig. 2a). To further confirm that Axl indeed positively regulates TNS2, we knocked down Axl expression in Axl-context cell, U2OS cell line, and also examined TNS2 and glucose import-related molecules by Western blot analysis. Expression of TNS2 was drastically down-regulated by shAxl co-transfection (Fig. 2b, lanes 2 and 4). Similarly, levels of p-Erk were downregulated by shAxl co-transfection. Because the transfected AXL and TNS2 contain neither their own promoters nor their own mRNA UTRs, the transfection effect apparently results from a post-translational mechanism (i.e., at the protein level). To distinguish ligand-induced Axl phosphorylation and ligand-independent Axl autophosphorylation, we utilized Gas6, the Axl ligand, to activate the Axl signaling pathway. Unfortunately, Gas6-induced Axl is not markedly upregulated in IRS1, p-Akt, or p-Erk after Gas6 treatment (despite Axl activation) (Fig. 2c) compared with Fig. 2a. We speculate that the effect observed was probably mediated through a ligand-independent pathway. Overall these results suggest that Axl positively regulates TNS2 expression. Since both Axl and TNS2 have been reported to be involved in glucose metabolism [2, 23], we also assessed IRS-1 expression and glucose uptake. The fold inductions of IRS-1 increased significantly upon TNS2 overexpression in cells (Fig. 2a lower panel, Additional file 2, and Fig. 2c). Consistently, knockdown of Axl expression abrogated TNS2 expression and significantly decreased cellular glucose import (Fig. 2d, upper left panel). Overexpression of Axl and/or TNS2 did not further enhance glucose uptake, likely because the endogenous levels of Axl and TNS2 expression were already sufficient for U2OS cells in terms of glucose uptake (Fig. 2d, lower panels).

\section{Axl directly phosphorylates TNS2 and mutation of TNS2 at Y483 attenuates cancer cell proliferation}

Reciprocal co-IP analysis was first performed to confirm the interaction between Axl and TNS2 in HEK293T cells (Fig. 3a and -Additional file 2). Axl has three auto-phosphorylation sites: Y779, Y821, and 


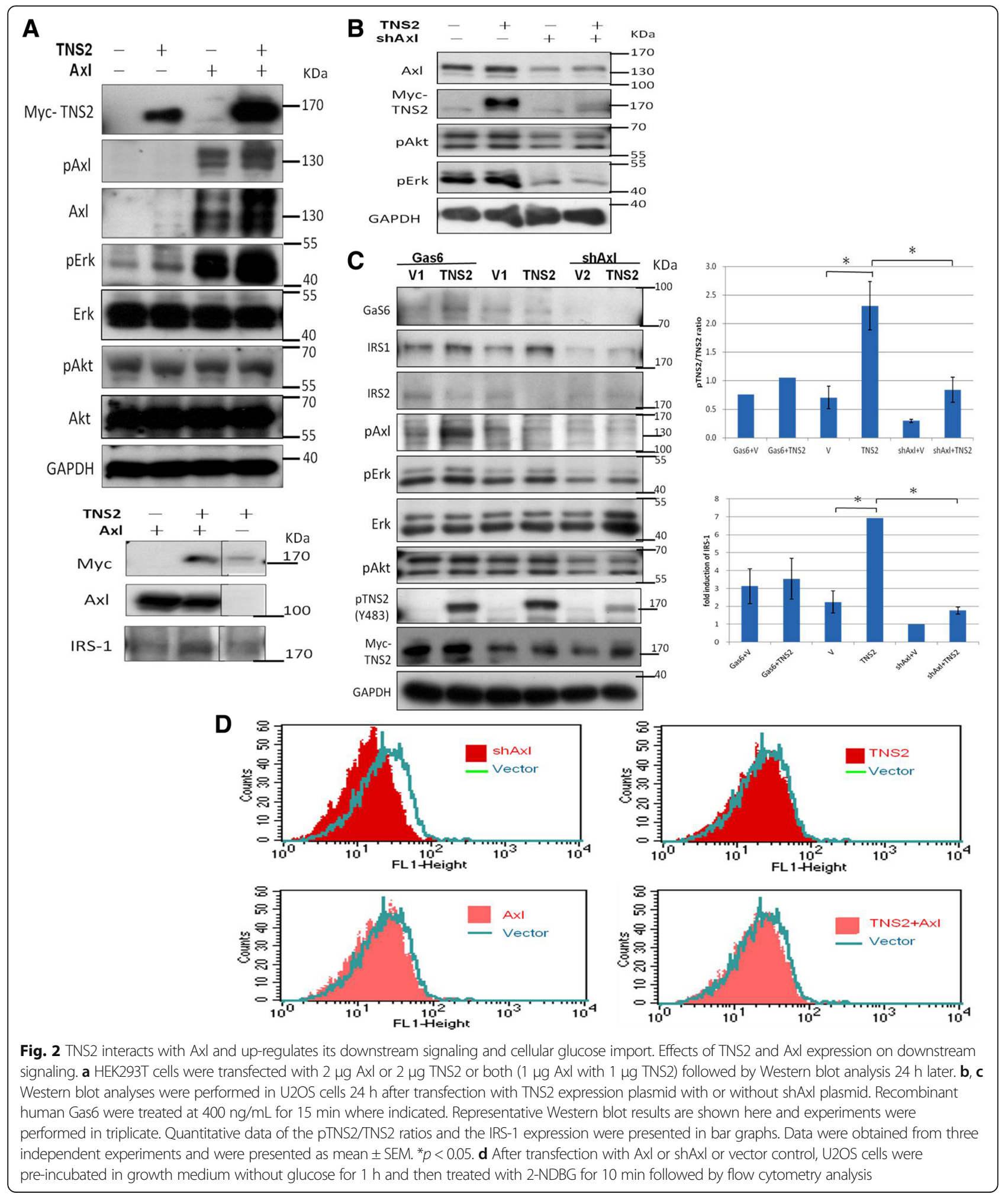

Y886. Y779 is important in Axl kinase activity [24]. We verified whether Y779 regulates phosphorylation of TNS2. The result demonstrated that all three sites are required for TNS2 phosphorylation and not just
Y779. The Y886F/Y821F double mutation did not affect the interaction between Axl and TNS2, whereas the kinase-death (KD) mutation (K567R) significantly abolished the interaction (Fig. 3a). Most importantly, 


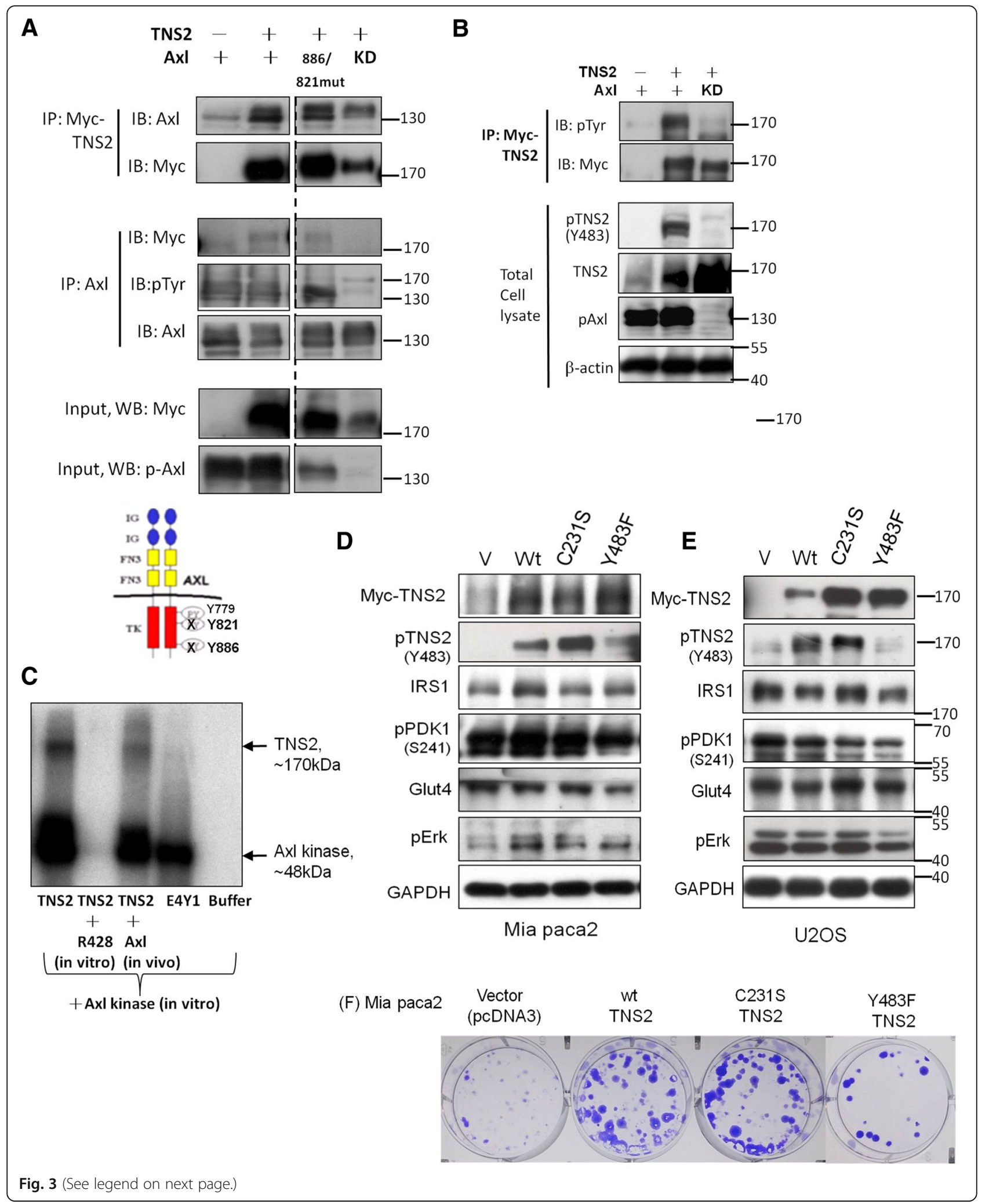


(See figure on previous page.)

Fig. $3 \mathrm{Axl}$ binds to and phosphorylates TNS2 at tyrosine 483 and regulates cancer cells proliferation. a To demonstrate the binding of TNS2 to Axl, HEK293T cells were transfected with TNS2 or Axl and lysed. TNS2 was co-immunoprecipitated (co-IP) by Myc and Axl. b To determine the effect of phosphorylation of Axl in activation of TNS2. HEK293T cells were transfected with TNS2 or Axl and lysed. TNS2 was IP by Myc, and blotted sequentially for phosphotyrosine (pTyr) and Myc. Total cell lysates were subjected to immunoblotting with related gene expression. c TNS2 is a substrate for AxI. TNS2 protein was pulled down from U2OS cells $24 \mathrm{~h}$ after being transfected with TNS2, followed by in vitro kinase assay. Poly(E4Y1) is an exogenous substrate for positive control. R428 is a specific Axl inhibitor. Effects of wild-type and the phosphatase-dead C231S and the phosphorylation mutant Y483F of TNS2 on the expression of various markers in Mia PaCa2 (d) and U2OS cells (e). All cells were transfected with various plasmids, respectively, and subjected to Western blot analysis $24 \mathrm{~h}$ later. $\mathbf{f}$ Effects of wild-type and the phosphatase-dead TNS2 (C231S) and the phosphorylation-dead TNS2 (Y483F) on colony formation in Mia PaCa2 cells. KD, kinase-death; Wt, wild type

we observed that Axl enhances TNS2 phosphorylation when the IP assay was conducted with p-tyrosine (Fig. 3b, upper panel) and western blot with anti-p-TNS2 (Fig. 3b, lower panel). We further demonstrated that Axl directly phosphorylates TNS2 by an in vitro kinase assay. The cell was transfected with Myc-TNS2 and then pulled down with Myc. The Myc-TNS2 was phosphorylated by the addition of Axl kinase in vitro, but its phosphorylation was completely abolished by the specific Axl inhibitor R428 (Fig. 3c, lanes 1 and 2). This result suggested that TNS2 is a substrate of Axl kinase. Moreover, it only slightly bound when we co-transfected Axl and TNS2 in vivo in the culture dish for the same assay.

According our data, the dysregulation of the Axl kinase is involved in phosphorylation of TNS2. To determine the certain phosphorylation site of TNS2, we constructed two candidate mutations of TNS2, TNS2-Cysteine 231 (C231S) and TNS2-tyrosine 483 (Y483F). In previous studies, TNS2(C231), a secondary P-loop Cys, has been shown to be crucial for the phosphatase activity of TNS2 [12, 25]. It has been reported that TNS2 (Y483) is a phosphorylation site for Src [26, 27]. According to the previous study, C231S is a phosphatase-dead mutant of TNS2 and Y483F is a phosphorylation-dead mutant of TNS2. Here, we assessed the potential involvement of several insulin-signaling molecules in both $\mathrm{Mia} \mathrm{PaCa}-2$ and U2OS cells, which express low levels of endogenous Axl. Phosphorylation of TNS2 was abolished in the Y483F mutant but not in the C231S mutant (Fig. 3d and e). We further measured the IRS1-associated signaling during dysregulation of TNS2. Expressions of glucose metabolism-related proteins, IRS-1, Glut4, and PDK1, were decreased in Mia PaCa-2 and U2OS cells expressing TNS2 (Y483F) compared with that in cells expressing TNS2 (wild type) (Fig. 3d and e). More importantly, the mutant Y483 site of TNS2 reflected less colony formation (Fig. 3f). The trend of colony-formation ability was consistent with the expression levels of IRS-1, Glut4, and PDK1 (Fig. 3d and f). Based on these findings, the expression level of TNS2 and its associated, IRS-1, Glut4 and PDK1 were found to be crucial for colony formation.
Clinical association of TNS2 and Axl expression in human pancreatic ductal adenocarcinomas

We performed tissue microarray immunohistochemical analysis on 33 paired human PDAC samples and adjacent non-tumor tissues. The clinicopathological features of the 33 patients were stratified into four stages: $30.3 \%$ $(11 / 33)$ stage I, 60.6\% (20/33) stage II, 0\% (0/33) stage III, and $6 \%(2 / 33)$ stage IV. There were no statistically significant differences (among all four groups, $p>0.05$ ) between early (IIa and earlier) and late stages (IIb and later) in the expression of Axl, TNS2, IRS1, and Glut4 proteins (Additional file 3). Consistent with the Western blot data, p-TNS2 and p-Axl were highly correlated $(p<$ 0.0005 , Table 1$)$. In the adjacent normal pancreatic tissues, p-TNS2 and p-Axl were highly expressed in the endocrine acinar cells but had low expression in the ductal epithelial cells. [4, 5] Representative micrographs of TNS2, Axl, and p-TNS2, p-Axl are shown in Figs. 1a and 4a. Quantitative analysis showed that the IHC-score of p-Axl and p-TNS2 in patient tumors was significantly greater than those of patient adjacent normal tissues (Fig. 4b and c). Consistent with TNS2 and Axl expression, the insulin signaling-related molecules, including - IRS-1 and Glut4 - had a similar trend in ductal areas (Additional file 4). Furthermore, statistical analysis of PDAC patients' tissue microarray IHC scoring showed that TNS2 was positively associated with the glucose metabolism-related IRS-1 and Glut4, the proliferation marker ki-67, the angiogenesis marker CD31, and the

Table 1 Correlation between Axl, p-Axl, TNS2, and p-TNS2, as examined using pancreatic tissue array slides

\begin{tabular}{lll}
\hline & $r$ & $p$ \\
\hline Tensin2 & & \\
Axl & 0.198 & 0.270 \\
pAxl (Y779) & 0.199 & 0.268 \\
pTensin2 (Y483) & -0.130 & 0.471 \\
pTensin2 & & \\
Axl & 0.069 & 0.702 \\
pAxl (Y779) & 0.629 & $0.000^{*}$ \\
Tensin2 (Y483) & -0.130 & 0.471 \\
\hline
\end{tabular}

The correlations were evaluated using the Pearson correlation test. Patient number $=33,{ }^{*} p>0.0005$ 


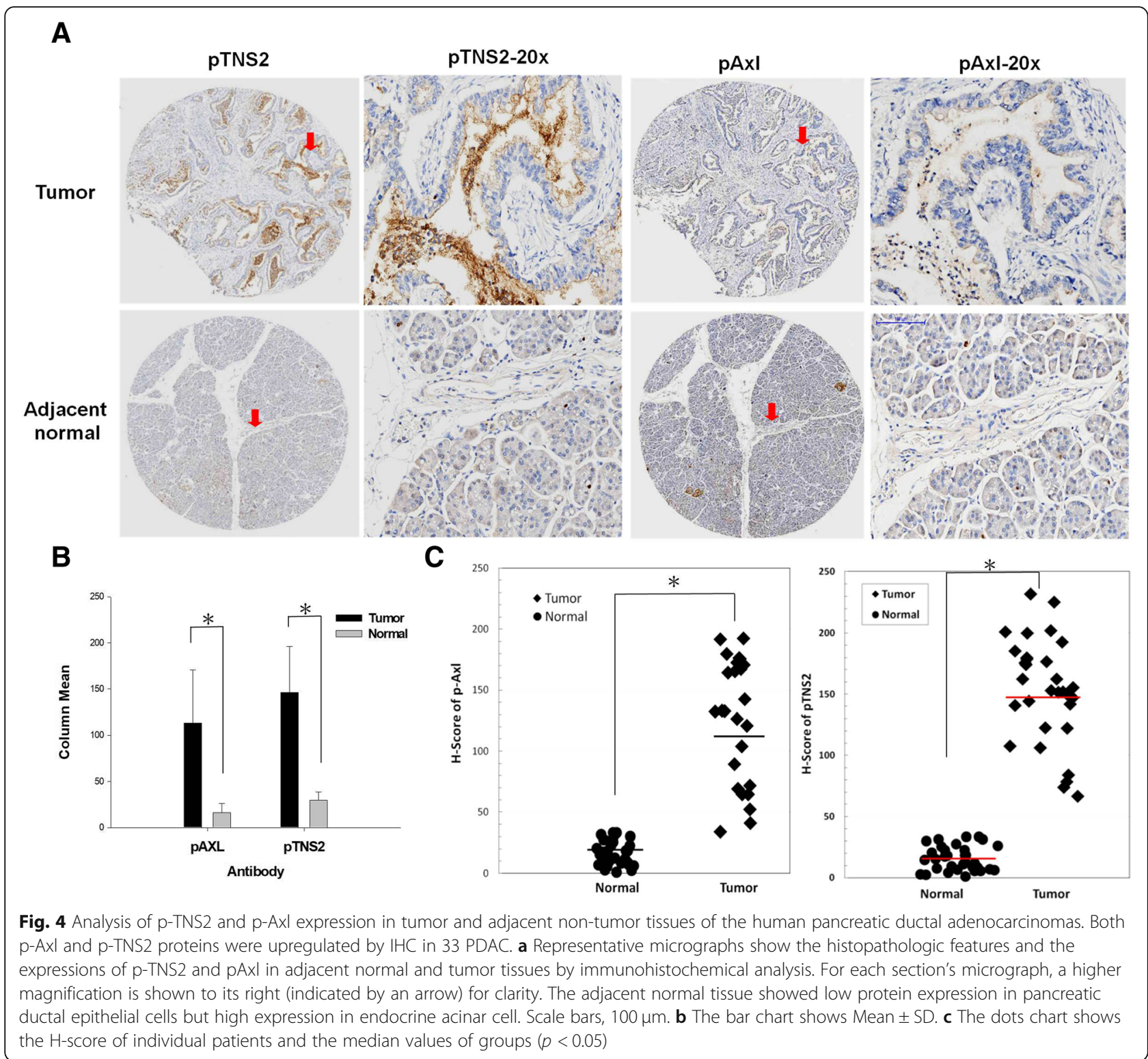

mesenchymal markers $\mathrm{N}$-cadherin and fibronectin (Table 2, left); in addition, Axl was positively associated with the mesenchymal marker fibronectin (Table 2, right). Similar analysis showed that p-TNS2 was positively associated with the cytoplasmic p-Akt, and that $\mathrm{p}$-Axl was positively associated with $\mathrm{N}$-cadherin, $\mathrm{p}$-Akt and IRS-1 (Table 3). These results suggested that the expression of TNS2 may involved in pancreatic cnacer metabolism, proliferation, angiogenesis, and epithelialmesenchymal transition (EMT) process.

\section{Discussion}

The receptor tyrosine kinase Axl is a well-known molecule that promotes tumor progression in various cancers. Cancer cells rewire their metabolism to promote cancer progression; one of the common features of this altered metabolism is increased glucose uptake and the conversion of glucose to lactate [28]. However, the function of Axl in cancer metabolism is not evident. Based on previous reports, surviving dormant cancer cells may over-express insulin-like growth factor-1 receptor (IGF-1R) during recurrence [29] and Axl may mediate different signals in high and low glucose situations [2, 30]. The results of our present study suggest that knockdown of Axl expression reduces glucose uptake and that Axl is related to high expression of glucose metabolism molecules, IRS-1, Glut4 and PDK1 in cancer tissues. We investigated the possible mechanism of Axl-regulated cancer metabolism through TNS2 expression. Additionally, we identified TNS2 as a substrate of Axl (Fig. 3c). 
Table 2 Correlation between Axl or TNS2, and various markers using pancreatic tissue arrays

\begin{tabular}{|c|c|c|}
\hline & $r$ & $p$ \\
\hline \multicolumn{3}{|l|}{ Tensin2 } \\
\hline Glut4 & 0.537 & $0.001^{*}$ \\
\hline IRS-1 & 0.335 & $0.043^{*}$ \\
\hline Ki-67 & 0.432 & $0.012^{*}$ \\
\hline CD31 & 0.435 & $0.011^{*}$ \\
\hline E-cadherin & 0.289 & 0.102 \\
\hline $\mathrm{N}$-cadherin & 0.452 & $0.008^{*}$ \\
\hline Fibronectin & -0.361 & $0.039^{*}$ \\
\hline Caspase3 & -0.083 & 0.644 \\
\hline pAkt (nuclei) & 0.297 & 0.093 \\
\hline pAkt (cytoplasm) & -0.029 & 0.872 \\
\hline pErk (nuclei) & 0.182 & 0.319 \\
\hline pErk (cytoplasm) & 0.229 & 0.200 \\
\hline \multicolumn{3}{|l|}{$A \times l$} \\
\hline Glut4 & 0.247 & 0.165 \\
\hline IRS-1 & 0.133 & 0.461 \\
\hline Ki-67 & 0.119 & 0.509 \\
\hline CD31 & 0.251 & 0.159 \\
\hline E-cadherin & 0.072 & 0.690 \\
\hline $\mathrm{N}$-cadherin & -0.168 & 0.350 \\
\hline Fibronectin & -0.345 & $0.049^{*}$ \\
\hline Caspase3 & 0.149 & 0.408 \\
\hline pAkt (nuclei) & 0.058 & 0.746 \\
\hline pAkt (cytoplasm) & 0.217 & 0.224 \\
\hline pErk (nuclei) & 0.186 & 0.307 \\
\hline pErk (cytoplasm) & 0.033 & 0.856 \\
\hline
\end{tabular}

The correlations were evaluated using the Pearson correlation test

Glut4 and IRS1 are involved in glycolysis; Ki-67, proliferation marker; CD31, angiogenesis marker; E-cadherin, epithelial marker; $\mathrm{N}$-cadherin and fibronectin, mesenchymal marker; Caspase 3, apoptosis marker. Patient number $=33,{ }^{*} p<0.05$

TNS2 region contains both an $\mathrm{SH} 2$ domain and a PTB domain, suggesting that an interaction with Axl might be mediated by phosphotyrosine. Axl enhanced TNS2 expression in Fig. 2a and kinase-dead of Axl abrogates TNS2 expression in Fig. 3b. According to these results, we suggested that Axl may regulate the stability of TNS2 through the interaction and phosphorylation of TNS2. The mechanism might involved in regulation of TNS2 degradation and stability of TNS2 expression. Unexpectedly, the results of the downstream molecules of Axl, p-Akt and p-Erk [31-33], were conflicting from cell culture and tissue specimens. Both p-TNS2 and p-Axl were positively associated with the cytoplasmic p-Akt but not p-Erk (Tables 2 and 3). However, there is no effect of overexpression of myc-TNS2 and Axl on p-Akt levels (Fig. 2a and c). The results of studies involving human embryonic
Table 3 Correlation between p-Axl or p-TNS2 and various markers using pancreatic tissue arrays

\begin{tabular}{|c|c|c|}
\hline & $r$ & $p$ \\
\hline \multicolumn{3}{|l|}{ pTensin2 } \\
\hline Glut4 & -0.037 & 0.082 \\
\hline IRS-1 & 0.003 & 0.988 \\
\hline Ki-67 & 0.106 & 0.556 \\
\hline CD31 & -0.073 & 0.683 \\
\hline E-cadherin & 0.079 & 0.661 \\
\hline $\mathrm{N}$-cadherin & 0.082 & 0.649 \\
\hline Fibronectin & 0.179 & 0.321 \\
\hline Caspase3 & 0.263 & 0.139 \\
\hline pAkt (nuclei) & -0.039 & 0.830 \\
\hline pAkt (cytoplasm) & 0.521 & $0.002^{*}$ \\
\hline pErk (nuclei) & 0.286 & 0.112 \\
\hline pErk (cytoplasm) & 0.018 & 0.919 \\
\hline \multicolumn{3}{|l|}{$p A x I$} \\
\hline Glut4 & -0.058 & 0.750 \\
\hline IRS-1 & 0.273 & 0.125 \\
\hline Ki-67 & 0.138 & 0.443 \\
\hline CD31 & 0.209 & 0.244 \\
\hline E-cadherin & 0.037 & 0.836 \\
\hline $\mathrm{N}$-cadherin & 0.437 & $0.011^{*}$ \\
\hline Fibronectin & 0.028 & 0.875 \\
\hline Caspase3 & 0.087 & 0.631 \\
\hline pAkt (nuclei) & 0.423 & $0.014^{*}$ \\
\hline pAkt (cytoplasm) & 0.749 & $0.000^{*}$ \\
\hline pErk (nuclei) & 0.211 & 0.246 \\
\hline pErk (cytoplasm) & 0.286 & 0.107 \\
\hline
\end{tabular}

The correlations were evaluated using the Pearson correlation test

Glut4 and IRS1 are involved in glycolysis; Ki-67, proliferation marker; CD31, angiogenesis marker; E-cadherin, epithelial marker; $\mathrm{N}$-cadherin and fibronectin are mesenchymal markers; Caspase 3, apoptosis marker. Patient number $=33,{ }^{*} p<0.05$

kidney $293 \mathrm{~T}$ (Fig. 2a), human osteosarcoma U2OS, and human pancreatic cancer specimens (Tables 2 and 3) are conflicting. Although Ara Koh et al. reported that IRS-1 regulates p-Akt in type 2 diabetes mellitus [12], its possible mechanism in cancer is yet to be elucidated. In addition, Cavet et al. reported that at high-glucose condition, Axl modulates p-Erk to vascular smooth muscle cell migration, whereas at low-glucose condition, Axl modulates p-Akt [2]. Both p-Akt and p-Erk are modulated among various tissues as well as different glucose conditions. In our data, we cultured all cells in high-glucose DMEM medium. However, we did not investigate the glucose condition and/or hypoxia conditons of the patients suffering from pancreatic cancer. We speculate that these results may related to the cell contexts of the cell lines and the high-glucose culture conditions we used. 
IRS-1 is a critical mediator in insulin signaling that regulates glucose uptake and PDK1-mediated glucose metabolism [34]. A recent report indicated a novel mechanism which TNS2 mediates IRS-1 degradation via dephosphorylation at IRS-1 Y612. This mechanism is different from the canonical insulin/IGF-1-induced IRS-1 degradation via phosphorylation at certain serine sites of IRS-1 in diabetes patients [12]. Several proteins have been found to interact with TNS2 through its C231 site of the PTPase domain $[8,25]$; this C231 mutation may abolish the tumorigenicity of breast and colon cancer cells [17]. The Oncomine reference database was used to explore the effects of TNS2 in cancer cells. Its role in various cancer types is not definitively established yet; moreover, TNS2's role in PDAC remains unknown (Additional file 5). In this study, however, we demonstrate for the first time that Axl directly binds to and phosphorylates TNS2 at Y483. The Y483 site is close to the PTPase domain of TNS2. The PTPase domain is critical for the de-phosphorylation of certain tyrosine kinases and for the tumor-suppressive functions of TNS2. The role of TNS2-Y483 in the insulin signaling pathway is not yet fully characterized. Our results show that the Y483 site is more important than the C231 site of TNS2 for regulation of IRS-1 expression and IRS-1-mediated signaling. By using tissue microarrays of pancreatic patients, we also demonstrate that in comparison to the adjacent normal parts of pancreaatic patients, cancer cells highly express both Axl (total form and the Y799-phosphorylated form) and TNS2 (total form and the Y483-phosphorylated form), as well as other molecules involved in glucose metabolism and cancer progression. In the future, it will be interesting to investigate whether Y483 phosphorylation of TNS2 plays a role in regulating IRS-1 and glucose metabolism with respect to the Warburg effect. Insulin resistance causes diminished glucose uptake not only in type 2 diabetes mellitus (DM2) but also in the brain in Alzheimer's disease (AD) [35]. IRS-1-mediated physiology is regulated by its several tyrosine residues and certain serine and threonine residues, which promote and reduce insulin signaling, respectively. Dysregulated phosphorylation of tyrosine and serine/threonine in IRS-1 may lead to the pathologic state of insulin resistance [36]. Recent studies indicate that phosphorylation of IRS-1 at S307 and/or at certain tyrosine residues can serve as a biomarker in peripheral exosomes, in both DM2 and AD patients [35, 37]. Once the effects of Axl on glucose uptake and/or glucose metabolism are established, novel strategies may be devised to target the molecules involved in these pathways, e.g., TNS2 and IRS-1, to improve the treatment for

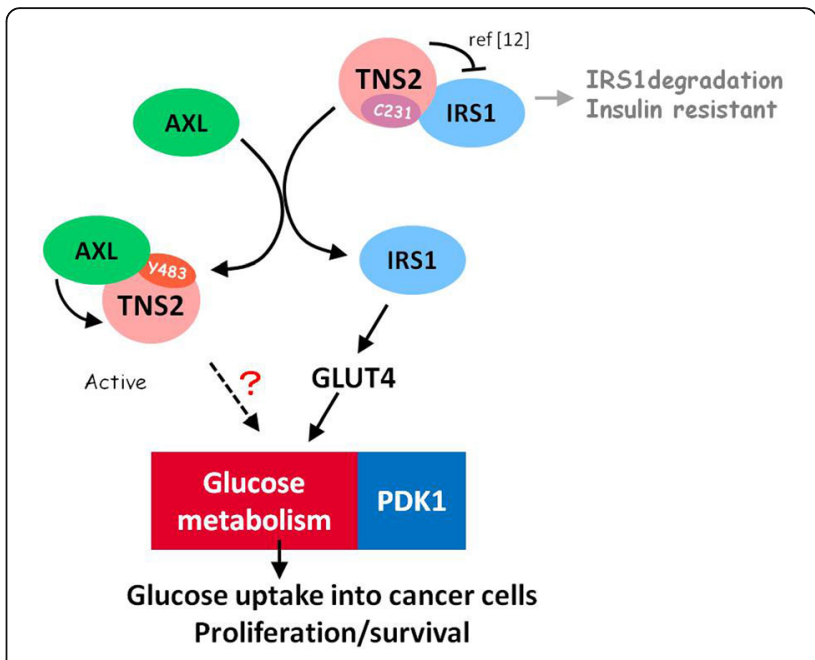

Fig. 5 Graphical abstract. Our results revealed a novel mechanism whereby Axl binds and phosphorylates TNS2 at Y483, releasing TNS2 from interacting with IRS-1, resulting in increased stability of IRS-1. Higher levels of IRS-1 may enhance cellular glucose uptake through up-regulation of Glut 4 and cell growth and survival. The two key enzymes of aerobic glycolysis, PDK1 was found to be up-regulated by the Axl/TNS2/IRS-1 cross-talk. The details of this regulatory cross-talk remain to be elucidated

PDAC and diabetes. We further speculate that the interaction of Axl and TNS2 could be a potential therapeutic target for the management of the IRS-1-associated cancer progression and other related diseases.

\section{Conclusions}

Our results revealed a novel mechanism whereby Axl binds and phosphorylates TNS2 at Y483, releasing TNS2 from interacting with IRS-1, resulting in increased stability of IRS-1 and the IRS-1 associated metabolism (Fig. 5). The interaction of Axl and TNS2 could serve as a potential therapeutic target for the management of the IRS-1-associated cancer progression and other related diseases. The Axl/TNS2/IRS-1 cross-talk may further potentially play a critical role in glucose metabolism of cancer cells.

\section{Additional files}

Additional file 1: The endogous expression of TNS2, p-Axl and Axl in five pancreatic cancer cell lines. Densityometric quantitative analyses of the TNS2 expression and pAxI/AxI ratio was performed in bar graphs. (PDF 117 kb)

Additional file 2: The original gel image of Fig. 3a. To demonstrate the binding of TNS2 to Axl, HEK293T cells were transfected with TNS2 or variant mutants of Axl and lysed. TNS2 was co-immunoprecipitated (co-IP) by Myc and Axl. K567R: Kinase-dead Axl (KD-Axl). (PDF 132 kb)

Additional file 3: Clinicopathological characteristics of the 33 pancreatic adenocarcinoma patients. The $\mathrm{H}$-score data are shown as mean \pm SD. (PDF $55 \mathrm{~kb}$ ) 
Additional file 4: Total TNS2 and total AXL expression in pancreatic cancer cells. Both proteins were detected by IHC in 33 PDAC. Representative micrographs show the histopathologic features and the expressions of TNS2, Axl, IRS-1, and GLUT4 in tumor and the adjacent normal tissues by immunohistochemical analysis. (PDF $164 \mathrm{~kb}$ )

Additional file 5: Expression of TNS2 (TENC1) in the Oncomine reference database. (PDF $148 \mathrm{~kb}$ )

\section{Abbreviations}

DLC: Deleted in liver cancer 1; FAK: Focal adhesion kinase; Gas6: Growth arrest specific protein 6; Glut4: glucose transporter type 4;

IP: Immunoprecipitation; IRS-1: Insulin receptor substrate-1; KD: Kinase-dead Mpl: Myeloproliferative leukemia; PDAC: Pancreatic ductal adenocarcinoma; PDK1: Pyruvate dehydrogenase kinase 1; PI3K: Phosphatidylinositol-3-kinase; PTB: Phosphotyrosine-binding; PTP: Protein tyrosine phosphatise; SH2: Src Homology 2; TNS2: Tensin 2

\section{Acknowledgements}

The pCMV-c-Myc-wild-type TNS2 plasmid is a gift from Drs. B. Dahlbäck and S. Hafizi of Lund University, Sweden. We thank the Pathology Core Laboratory of NHRI for providing services.

\section{Funding}

This work was supported by National Health Research Institutes, Taiwan (CA-106-PP-14).

\section{Availability of data and materials}

There are not dataset or database within the article.

\section{Authors' contributions}

LCC and Dr. ANC were responsible for the study concept and design. Dr. YLC performed the tissue microarray and statistical analyses. LCC performed the experiments. Dr. CYC designed the plasmids. LCC, Dr. ANC, Dr. AYL and Dr. JSH were responsible for the discussion of the experimental results and interpretation. LCC and Dr. SEC were responsible for writing and finalizing the manuscript. All authors read and approved the final manuscript.

\section{Ethics approval and consent participate}

All procedures involving patient IHC tissues were delinked anonymously for protection and approved by the Institutional Review Board (IRB) of Cardinal Tien Hospital (CTH-101-3-5-054).

\section{Consent for publication}

Not applicable.

\section{Competing interests}

The authors declare that they have no competing interests.

\section{Publisher's Note}

Springer Nature remains neutral with regard to jurisdictional claims in published maps and institutional affiliations.

\section{Author details}

${ }^{1}$ Graduate Institute of Life Sciences, National Defense Medical Center, Taipei, Taiwan. ${ }^{2}$ Department of Pathology, Cardinal Tien Hospital, School of Medicine, Fu-Jen Catholic University, New Taipei City, Taiwan. ${ }^{3}$ National Institute of Cancer Research, National Health Research Institutes, 35 Keyan Road, Zhunan, Miaoli County 350, Taiwan, Republic of China. ${ }^{4}$ Department of Pediatrics, Kaohsiung Medical University Hospital, Kaohsiung, Taiwan.

Received: 30 July 2018 Accepted: 15 August 2018

Published online: 12 November 2018

\section{References}

1. Weinger JG, Brosnan CF, Loudig O, Goldberg MF, Macian F, et al. Loss of the receptor tyrosine kinase $A x l$ leads to enhanced inflammation in the CNS and delayed removal of myelin debris during experimental autoimmune encephalomyelitis. J Neuroinflammation. 2011;8:49.
2. Cavet ME, Smolock EM, Ozturk OH, World C, Pang J, et al. Gas6-Axl receptor signaling is regulated by glucose in vascular smooth muscle cells. Arterioscler Thromb Vasc Biol. 2008;28:886-91.

3. Bauer T, Zagórska A, Jurkin J, Yasmin N, Köffel R, et al. Identification of Axl as a downstream effector of TGF- $\beta 1$ during Langerhans cell differentiation and epidermal homeostasis. J Exp Med. 2012;209:2033-47.

4. Song X, Wang H, Logsdon CD, Rashid A, Fleming JB, et al. Overexpression of receptor tyrosine kinase $\mathrm{Axl}$ promotes tumor cell invasion and survival in pancreatic ductal adenocarcinoma. Cancer. 2011;117:734-43.

5. Leconet W, Larbouret C, Chardes T, Thomas G, Neiveyans M, et al. Preclinical validation of AXL receptor as a target for antibody-based pancreatic cancer immunotherapy. Oncogene. 2014;33:5405-14.

6. Lo SH. Tensin. The International Journal of Biochemistry \&amp. Cell Biology. 2004;36:31-4.

7. Liu BA, Engelmann BW, Nash PD. The language of SH2 domain interactions defines phosphotyrosine-mediated signal transduction. FEBS Lett. 2012;586: 2597-605.

8. Hafizi S, Alindri F, Karlsson R, Dahlback B. Interaction of Axl receptor tyrosine kinase with C1-TEN, a novel C1 domain-containing protein with homology to tensin. Biochem Biophys Res Commun. 2002;299:793-800.

9. Hafizi S, Sernstad E, Swinny JD, Gomez MF, Dahlback B. Individual domains of Tensin2 exhibit distinct subcellular localisations and migratory effects. Int Biochem Cell Biol. 2010;42:52-61.

10. Moon KD, Zhang X, Zhou Q, Geahlen RL. The protein-tyrosine kinase Syk interacts with the C-terminal region of tensin2. Biochim Biophys Acta. 2012 1823:199-205.

11. Jung AS, Kaushansky A, Macbeath G, Kaushansky K. Tensin2 is a novel mediator in thrombopoietin (TPO)-induced cellular proliferation by promoting Akt signaling. Cell Cycle. 2011;10:1838-44.

12. Koh $\mathrm{A}$, Lee $M N$, Yang $Y R$, Jeong $H$, Ghim J, et al. C1-ten is a protein tyrosine phosphatase of insulin receptor substrate 1 (IRS-1), regulating IRS-1 stability and muscle atrophy. Mol Cell Biol. 2013:33:1608-20.

13. Chan LK, Ko FC, Ng IO, Yam JW. Deleted in liver cancer 1 (DLC1) utilizes a novel binding site for Tensin2 PTB domain interaction and is required for tumor-suppressive function. PLoS One. 2009;4:e5572.

14. Dai K, Liao S, Zhang J, Zhang X, Tu X. Solution structure of tensin2 SH2 domain and its phosphotyrosine-independent interaction with DLC-1. PLoS One. 2011;6:e21965

15. Kawai K, Kitamura SY, Maehira K, Seike J, Yagisawa H. START-GAP1/DLC1 is localized in focal adhesions through interaction with the PTB domain of tensin2. Adv Enzym Regul. 2010;50:202-15.

16. Goudarzi S, Smith LJ, Schutz S, Hafizi S. Interaction of DISC1 with the PTB domain of Tensin2. Cell Mol Life Sci. 2013:70:1663-72.

17. Hong SY, Shih YP, Sun P, Hsieh WJ, Lin WC, et al. Down-regulation of tensin2 enhances tumorigenicity and is associated with a variety of cancers. Oncotarget. 2016;7:38143-53

18. Daemen A, Peterson D, Sahu N, McCord R, Du X, et al. Metabolite profiling stratifies pancreatic ductal adenocarcinomas into subtypes with distinct sensitivities to metabolic inhibitors. Proc Natl Acad Sci. 2015:112:E4410-7.

19. Tan Z, Xie N, Cui H, Moellering DR, Abraham E, et al. Pyruvate dehydrogenase kinase 1 participates in macrophage polarization via regulating glucose metabolism. J Immunol. 2015;194(12):6082-9.

20. EM P-MD, Curtis AM, Goel G, Lauterbach MA, Sheedy FJ, et al. Pyruvate kinase $\mathrm{M} 2$ regulates Hif-1alpha activity and IL-1beta induction and is a critical determinant of the warburg effect in LPS-activated macrophages. Cell Metab. 2015;21:65-80.

21. Hong CC, Lay JD, Huang JS, Cheng AL, Tang JL, et al. Receptor tyrosine kinase $A X L$ is induced by chemotherapy drugs and overexpression of $A X L$ confers drug resistance in acute myeloid leukemia. Cancer Lett. 2008;268: $314-24$

22. Hafizi S, Ibraimi F, Dahlbäck B. C1-TEN is a negative regulator of the Akt/PKB signal transduction pathway and inhibits cell survival, proliferation, and migration. FASEB J. 2005;19:971-3.

23. Shi Y, Wang J, Chandarlapaty S, Cross J, Thompson C, et al. PTEN is a protein tyrosine phosphatase for IRS1. Nat Struct Mol Biol. 2014;21:522-7.

24. Korshunov VA. Axl-dependent signalling: a clinical update. Clin Sci (Lond). 2012;122:361-8.

25. Hafizi S, Gustafsson A, Oslakovic C, Idevall-Hagren O, Tengholm A, et al. Tensin2 reduces intracellular phosphatidylinositol 3,4,5-trisphosphate levels at the plasma membrane. Biochem Biophys Res Commun. 2010; 399:396-401. 
26. Ferrando IM, Chaerkady R, Zhong J, Molina H, Jacob HK, et al. Identification of targets of c-Src tyrosine kinase by chemical complementation and phosphoproteomics. Mol Cell Proteomics. 2012;11:355-69.

27. Renes J, Mariman E. Application of proteomics technology in adipocyte biology. Mol BioSyst. 2013;9:1076-91.

28. Liberti MV, Locasale JW. The Warburg effect: how does it benefit Cancer cells? Trends Biochem Sci. 2016;41:211-8.

29. Rajbhandari N, Lin WC, Wehde BL, Triplett AA, Wagner KU. Autocrine IGF1 signaling mediates pancreatic tumor cell dormancy in the absence of oncogenic drivers. Cell Rep. 2017;18:2243-55.

30. Lee C-H, Shieh Y-S, Hsiao F-C, Kuo F-C, Lin C-Y, et al. High glucose induces human endothelial dysfunction through an Axl-dependent mechanism. Cardiovasc Diabetol. 2014;13:1-13.

31. Salian-Mehta S, Xu M, Wierman ME. AXL and MET crosstalk to promote gonadotropin releasing hormone $(\mathrm{GnRH})$ neuronal cell migration and survival. Mol Cell Endocrinol. 2013;374:92-100.

32. Huang JS, Cho CY, Hong CC, Yan MD, Hsieh MC, et al. Oxidative stress enhances Axl-mediated cell migration through an Akt1/Rac1-dependent mechanism. Free Radic Biol Med. 2013;65:1246-56.

33. Liu L, Greger J, Shi H, Liu Y, Greshock J, et al. Novel mechanism of lapatinib resistance in HER2-positive breast tumor cells: activation of AXL. Cancer Res. 2009:69:6871-8

34. Zha J, Lackner MR. Targeting the insulin-like growth factor receptor-1R pathway for Cancer therapy. Clin Cancer Res. 2010;16:2512-7.

35. Kapogiannis D, Boxer A, Schwartz JB, Abner EL, Biragyn A, et al. Dysfunctionally phosphorylated type 1 insulin receptor substrate in neuralderived blood exosomes of preclinical Alzheimer's disease. FASEB J. 2015;29: 589-96.

36. Hançer NJ, Qiu W, Cherella C, Li Y, Copps KD, et al. Insulin and metabolic stress stimulate multisite serine/threonine phosphorylation of insulin receptor substrate 1 and inhibit tyrosine phosphorylation. J Biol Chem. 2014;289:12467-84.

37. Bassil F, Canron MH, Vital A, Bezard E, Li Y, et al. Insulin resistance and exendin4 treatment for multiple system atrophy. Brain. 2017;140(5):1420-36.

Ready to submit your research? Choose BMC and benefit from:

- fast, convenient online submission

- thorough peer review by experienced researchers in your field

- rapid publication on acceptance

- support for research data, including large and complex data types

- gold Open Access which fosters wider collaboration and increased citations

- maximum visibility for your research: over $100 \mathrm{M}$ website views per year

At $\mathrm{BMC}$, research is always in progress.

Learn more biomedcentral.com/submissions 